\title{
Constraining Transversity and Nucleon Transverse-polarization Structure Through Polarized-proton Collisions at STAR
}

\author{
Jim Drachenberg ${ }^{* \dagger}$ \\ Valparaiso University \\ E-mail: Jim.Drachenberg@valpo.edu
}

\begin{abstract}
Current knowledge of nucleon transverse-polarization structure comes from measurements of transverse single-spin asymmetries (SSAs) from semi-inclusive deep inelastic scattering (SIDIS). These measurements, combined with those of $e^{+} e^{-}$collisions, have allowed the first extractions of transversity with limited constraints at higher values of Bjorken- $x$. One avenue to enrich understanding over a different kinematic range is jet+hadron and di-hadron production from polarizedproton collisions. Through these channels, the STAR detector at RHIC has for the first time observed SSAs due to the effects of transversity coupled to the Collins and interference fragmentation functions (IFFs) in polarized-proton collisions at $\sqrt{s}=200$ and $500 \mathrm{GeV}$. In addition to transversity, the distribution of pions within jets may also provide a window into gluon linear polarization. Furthermore, the comparison of all asymmetry moments at $200 \mathrm{GeV}$ and 500 $\mathrm{GeV}$ may yield insight into longstanding theoretical questions concerning evolution, universality, and factorization breaking in non-collinear formulations of pQCD. Preliminary results from the jet+hadron and di-hadron analyses at $\sqrt{s}=200$ and $500 \mathrm{GeV}$ will be presented, including the first observations of transversity effects in polarized-proton collisions and the first-ever measurements offering constraints on models involving gluon linear polarization.
\end{abstract}

XXIII International Workshop on Deep-Inelastic Scattering

27 April - May 12015

Dallas, Texas

\footnotetext{
* Speaker.

${ }^{\dagger}$ for the STAR Collaboration.
} 


\section{Introduction}

The momentum structure of the nucleon at leading-twist can be described by three parton distribution functions (PDFs): the unpolarized parton distribution, $f(x)$; the helicity parton distribution, $\Delta f(x)$; and the transversity distribution, $h_{1}(x)$ [1]. Of the three, transversity, which describes the transverse polarization of quarks inside a transversely polarized nucleon, has proven the most difficult to probe, due to its chiral-odd nature. Many advances in understanding transversity have been made through the study of azimuthal transverse single-spin asymmetries, $A_{U T}$. Such studies in polarized-proton collisions present a challenge and an opportunity. To account for nonzero $A_{U T}$ from high- $p_{T}$ hadroproduction (e.g. Ref. [2]) one is challenged to understand pQCD beyond the collinear formulation at leading twist [3]. By so doing, one gains the opportunity for insight into the transverse polarization structure of the nucleon.

Two approaches that can generate nonzero $A_{U T}$ in $\mathrm{pQCD}$ are to formulate collinear pQCD to account for higher twist multi-parton correlators (twist-3 formalism) [4, 5] or to formulate pQCD to account for intrinsic transverse momentum dependence (TMD formalism) [6]. In the twist-3 formalism one can obtain asymmetries, in principle, from both the initial state and the fragmentation functions (e.g. Ref. [7]). Similarly, in the TMD formalism one can obtain asymmetries, in principle, from both the PDFs (the so-called "Sivers effect") [6] and the fragmentation functions, e.g. the socalled "Collins effect" [8]. Furthermore, it has been shown that the intrinsic transverse momentum integrals of the TMD functions are closely related to the twist-3 functions (e.g. Ref. [9]).

Over the past decade, experiments in semi-inclusive deep-inelastic scattering (SIDIS) have provided the first measurements of TMD observables (e.g. Ref. [10]). These, combined with independent measurements of the Collins fragmentation function by $e^{+} e^{-}$experiments [11], have enabled the first extractions of the transversity PDF (e.g. Ref. [12]). The kinematic limitations of current datasets leave the transversity extractions relatively imprecise for $x \gtrsim 0.2$.

One avenue to enrich understanding of nucleon spin structure is through jet production from high-energy polarized-proton collisions [13]. By measuring the spin-dependent, azimuthal asymmetry in the jet production $\left(A_{U T}^{\sin \phi_{S}}\right)$, one can access the twist-3 correlation function, related to the Sivers function. Additionally, by measuring different spin-dependent, azimuthal modulations in the distribution of hadrons within a jet $\left(A_{U T}^{\sin \left(\phi_{S}-\phi_{H}\right)}\right.$ or $\left.A_{U T}^{\sin \left(\phi_{S}-2 \phi_{H}\right)}\right)$, one can gain sensitivity to transversity or the gluon-analogue to transversity (sensitive to gluon linear polarization) coupling to spin-dependent Collins or "Collins-like" [14] fragmentation functions, respectively. Similarly to the Collins effect, one can also access transversity coupled to polarized "interference fragmentation functions" (IFF) through spin-dependent, azimuthal asymmetries in the relative orientation of two hadrons from the same parton (e.g. Refs. [15, 16]). While IFFs survive in the leading-twist, collinear formulation of pQCD with factorization expected to hold, the Collins effect depends upon TMD-factorization that is broken, in general, for high- $p_{T}$ hadroproduction [17]. Thus, by studying both Collins and IFF asymmetries for overlapping kinematics, one opens the possibility to enlighten deep theoretical questions, such as TMD factorization-breaking and universality.

The STAR detector [18] at RHIC has seen the first signatures of transversity in polarizedproton collisions from charged-pion Collins [19] and IFF [20] asymmetries at $|\eta|<1$ from 2.4 $\mathrm{pb}^{-1}$ at $\sqrt{s}=200 \mathrm{GeV}$ collected in 2006. Motivated in large part to improve the precision of these measurements, in 2012, STAR integrated $22 \mathrm{pb}^{-1}$ of luminosity from $p^{\uparrow}+p$ at $\sqrt{s}=200$ 

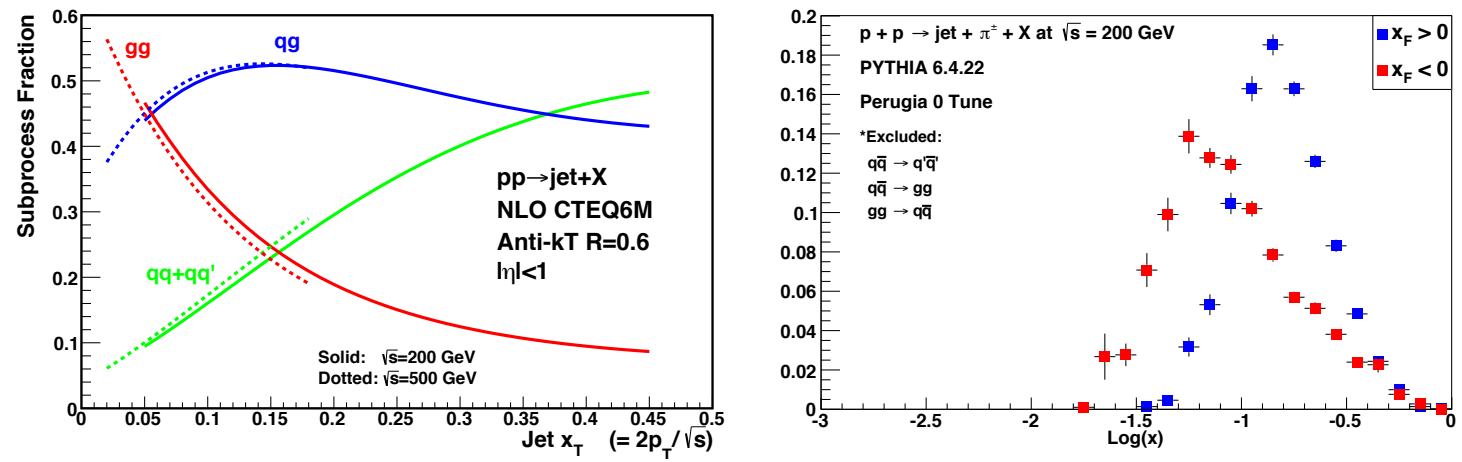

Figure 1: (left) Subprocess fractions and (right) unpolarized quark $x$ distribution for STAR jet production. The fractions of NLO cross section [21] are shown for quark-quark, gluon-gluon, or quark-gluon interactions and presented as functions of jet $x_{T}$ across a range of $5<p_{T}<45 \mathrm{GeV} / c$ for collision energies of $\sqrt{s}=200$ $\mathrm{GeV}$ and $\sqrt{s}=500 \mathrm{GeV}$. The unpolarized quark $x$ distribution is shown for jets with $p_{T}>10 \mathrm{GeV} / c$ for $\sqrt{s}=200 \mathrm{GeV}$ and separately for scattering forward $\left(x_{F}>0\right)$ and backward $\left(x_{F}<0\right)$ relative to the polarized beam.

$\mathrm{GeV}$ with $63 \%$ polarization. Furthermore, in 2011 STAR integrated $25 \mathrm{pb}^{-1}$ of luminosity from $p^{\uparrow}+p$ at $\sqrt{s}=500 \mathrm{GeV}$ with $53 \%$ polarization. This dataset allows the first measurements of these asymmetries at $\sqrt{s}=500 \mathrm{GeV}$, including the first-ever measurement of the "Collins-like" asymmetry, with sensitivity to gluonic subprocesses enhanced relative to $\sqrt{s}=200 \mathrm{GeV}$ (Fig. 1). Comparison of all asymmetry modulations across $\sqrt{s}=200$ and $500 \mathrm{GeV}$ is expected to extend the current knowledge of these effects to broader kinematics as well as inform questions about the evolution of transversity and the TMD functions.

\section{Analysis}

The present data were collected with a minimum-bias trigger (VPDMB), requiring a coincidence in STAR's vertex position detector (VPD) [22], as well as with "jet-patch" triggers, requiring patches of energy in STAR's barrel (BEMC) and endcap (EEMC) electromagnetic calorimeters [18]. Jets are reconstructed using the "anti- $k_{T}$ " algorithm [23] with a radius of 0.6 for $\sqrt{s}=200$ $\mathrm{GeV}$ or 0.5 for $\sqrt{s}=500 \mathrm{GeV}$ and utilize energy deposition in the BEMC and EEMC as well as charged-particle tracks from STAR's time projection chamber (TPC) [18].

Descriptions of the analysis techniques and simulation studies for the jet measurements are given in Refs. [24, 25, 26], while those for the IFF measurements are given in Refs. [20, 27, 28]. For jets, the dominant systematic uncertainties arise from jets reconstructed at the detector level that fail to match to one at the parton-jet level. Additional systematic uncertainties come from the contamination of kaons, protons, and electrons to the charged-pion signal; trigger bias; the "leakthrough" of competing effects coupling to non-uniform detector acceptance; uncertainties from calorimeter gains, efficiencies, and response to charged hadrons; tracking efficiency; and Monte Carlo simulation statistics. Measured asymmetries for $\sqrt{s}=500 \mathrm{GeV}$ are corrected for smearing due to finite azimuthal-angle resolution, while those for $\sqrt{s}=200 \mathrm{GeV}$ account for this effect with a systematic uncertainty. 

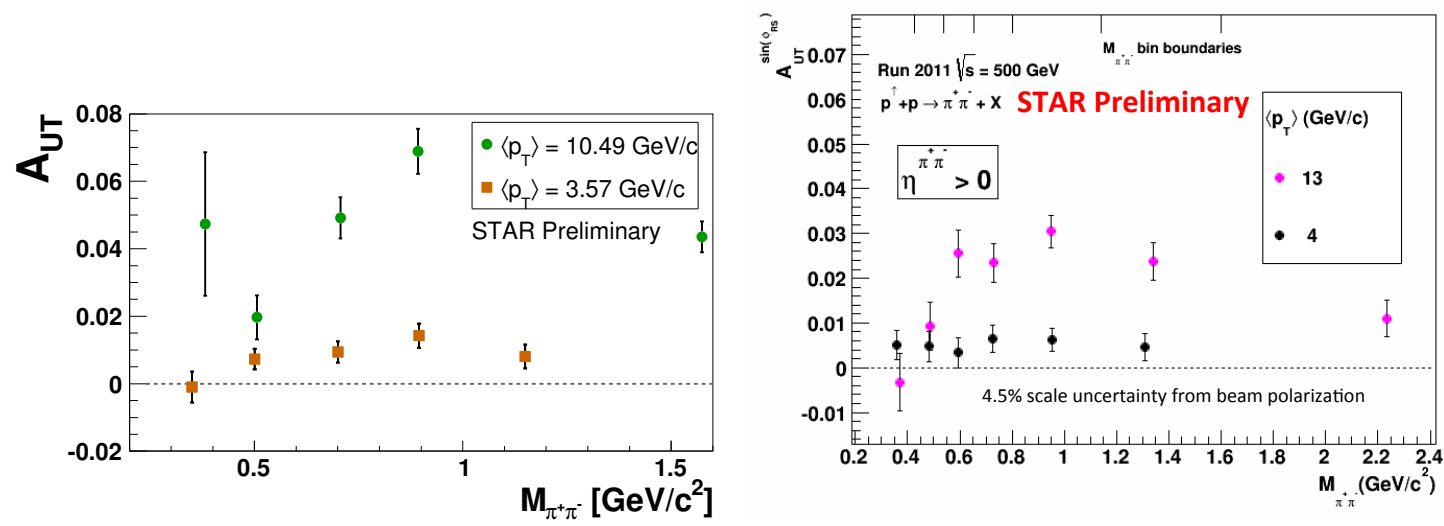

Figure 2: STAR preliminary charged-pion IFF asymmetries for (left) $\sqrt{s}=200 \mathrm{GeV}$ [28] and (right) $\sqrt{s}=500 \mathrm{GeV}$ [27]. Asymmetries are shown as functions of two-pion invariant mass for two bins of twopion $p_{T}$. For both $\sqrt{s}=200 \mathrm{GeV}$ and $\sqrt{s}=500 \mathrm{GeV}$, the asymmetries show qualitatively similar behavior with a strong dependence upon $p_{T}$.

\section{Results}

Figure 2 shows STAR preliminary charged-pion IFF asymmetries for $\sqrt{s}=200 \mathrm{GeV}$ [28] (collected in 2012) and $\sqrt{s}=500 \mathrm{GeV}$ [27] (collected in 2011) as functions of two-pion invariant mass, $M_{\pi^{+} \pi^{-}}$. The asymmetries are each presented for $\eta_{\pi^{+} \pi^{-}}>0$ (relative to the polarized beam) in two bins of two-pion $p_{T}$. For both $\sqrt{s}=200 \mathrm{GeV}$ and $\sqrt{s}=500 \mathrm{GeV}$, the asymmetries show qualitatively similar behavior, though the data have not yet been robustly examined for a quantitative comparison. The asymmetries show a strong dependence upon $p_{T}$ and a slower dependence upon invariant mass.

Figure 3 shows STAR preliminary charged-pion Collins asymmetries for $\sqrt{s}=200 \mathrm{GeV}$ and $\sqrt{s}=500 \mathrm{GeV}$ as functions of $z=p_{\pi} / p_{\text {jet }}$. The asymmetries are each presented for $\eta_{\text {jet }}>0$ (relative to the polarized beam). Selection criteria for the $\sqrt{s}=200 \mathrm{GeV}$ and $\sqrt{s}=500 \mathrm{GeV}$ datasets are chosen so that the two sets have roughly the same $x_{T}$. For the $\sqrt{s}=200 \mathrm{GeV}$ data, jets are required to have $p_{T}>10 \mathrm{GeV} / c$ with $\left\langle p_{T}\right\rangle=12.9 \mathrm{GeV} / c$; while for the $\sqrt{s}=500 \mathrm{GeV}$ data, jets are required to have $22.7<p_{T}<55 \mathrm{GeV} / c$ with $\left\langle p_{T}\right\rangle=31.0 \mathrm{GeV} / c$. It is expected that the datasets will have sensitivity across a range of $0.1 \lesssim x \lesssim 0.3$ (Fig. 1 and Ref. [26]). In order to ensure further that the two datasets span the same kinematic phase space, the data are compared for two different sets of restrictions on the minimum hadron radius, $\Delta R=\sqrt{\left(\eta_{\mathrm{jet}}-\eta_{\pi}\right)^{2}+\left(\phi_{\mathrm{jet}}-\phi_{\pi}\right)^{2}}$. To ensure robust determination of the azimuthal orientation of the pion about the jet axis, $\Delta R$ is required to be above a minimum threshold. However, such a cut also restricts the sampled kinematic phase space, since

$$
j_{T, \min } \approx z \times \Delta R_{\min } \times\left\langle p_{T, \text { jet }}\right\rangle,
$$

where $j_{T}$ is the pion transverse momentum measured relative to the jet axis. Thus, in Fig. 3 , the data are presented for two sets of $\Delta R$ cuts: (top) $\Delta R>0.1$ for $\sqrt{s}=200 \mathrm{GeV}$ and $\Delta R>0.04$ for $\sqrt{s}=500 \mathrm{GeV}$ and (bottom) $\Delta R>0.25$ for $\sqrt{s}=200 \mathrm{GeV}$ and $\Delta R>0.1$ for $\sqrt{s}=500 \mathrm{GeV}$. For the more restrictive $\Delta R$ threshold shown in the bottom panel, the asymmetries are consistent with 


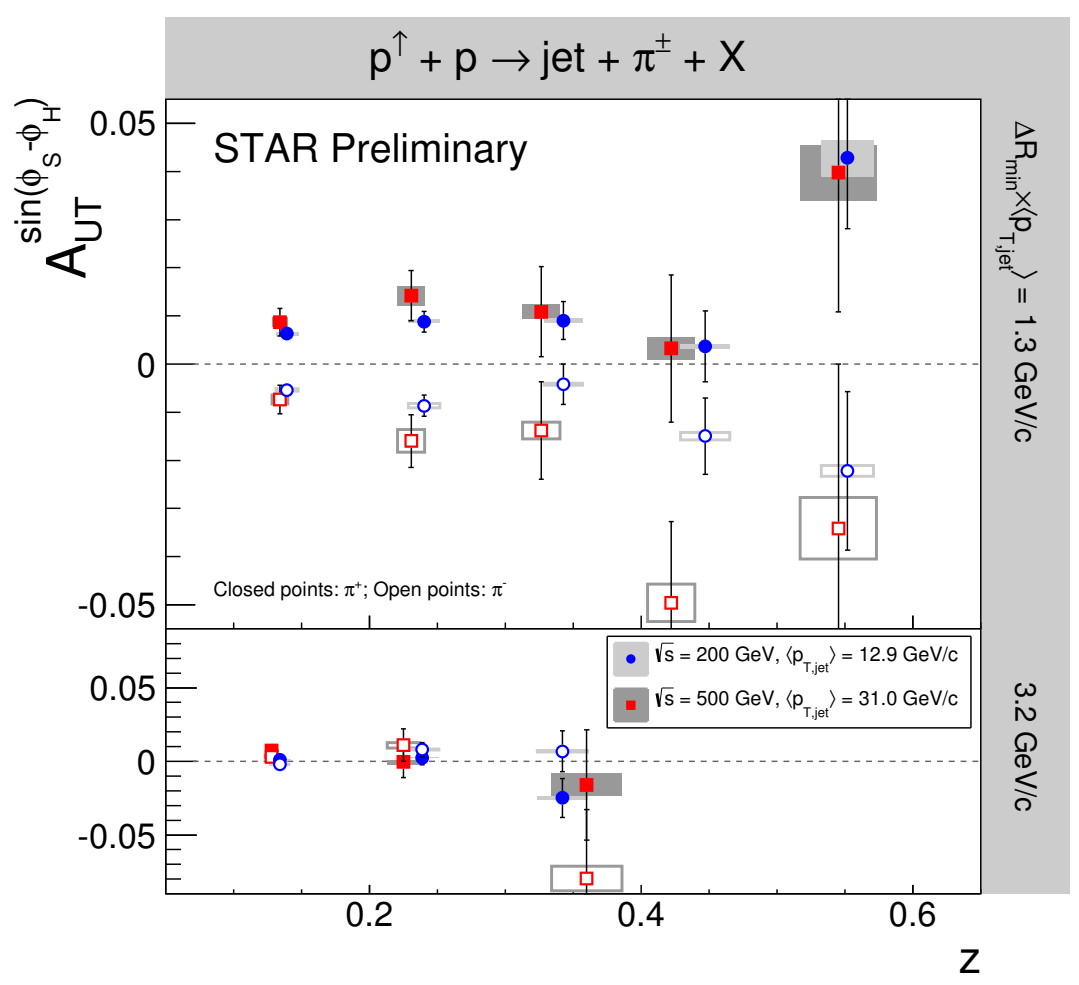

Figure 3: STAR preliminary charged-pion Collins asymmetries for $\sqrt{s}=200 \mathrm{GeV}$ and $\sqrt{s}=500 \mathrm{GeV}$ $[25,29,26]$. Asymmetries are shown as functions of pion $z$ for two bins of $\Delta R_{\min } \times\left\langle p_{T, \text { jet }}\right\rangle$. Within the current precision, STAR Collins asymmetries are consistent with $x_{T}$ scaling.

zero. In contrast, when the $\Delta R$ threshold is relaxed, as shown in the top panel, the asymmetries for both $\sqrt{s}=200 \mathrm{GeV}$ and $\sqrt{s}=500 \mathrm{GeV}$ are nonzero with positive asymmetries for $\pi^{+}$and negative asymmetries for $\pi^{-}$. This dependence on $\Delta R_{\min }$ demonstrates a strong dependence upon pion $j_{T}$ that can be seen explicitly for the $\sqrt{s}=200 \mathrm{GeV}$ data in Ref. [26]. Furthermore, within the current precision, the preliminary asymmetries are consistent with $x_{T}$ scaling between $\sqrt{s}=200 \mathrm{GeV}$ and $\sqrt{s}=500 \mathrm{GeV}$.

As can be seen in Refs. [24, 25], at $\sqrt{s}=500 \mathrm{GeV}$ the inclusive jet asymmetry, $A_{U T}^{\sin \phi_{S}}$, sensitive to the twist-3 correlation function $[4,5]$ (related to the Sivers function [6]), is consistent with zero across the range of $6<p_{T, \text { jet }}<55 \mathrm{GeV} / c$. This is consistent with previous measurements at $\sqrt{s}=200 \mathrm{GeV}$ [30] but with improved limits and enhanced sensitivity to gluonic subprocesses (Fig. 1). In addition, the Collins-like asymmetry, $A_{U T}^{\sin \left(\phi_{S}-2 \phi_{H}\right)}$, sensitive to linearly polarized gluons is measured for the first time and found to be consistent with zero, well below the maximally allowed projections of $\approx 2 \%$ [13]. These data should enable the first experimental constraints on gluon linear polarization.

\section{Conclusions}

For the first time, the effects of transversity in polarized-proton collisions are observed in 
charged-pion IFF and Collins asymmetries at both $\sqrt{s}=200 \mathrm{GeV}$ and $\sqrt{s}=500 \mathrm{GeV}$. At the current precision, the preliminary Collins asymmetries are consistent with $x_{T}$ scaling. The STAR data are expected to probe an $x$-range complementary to existing data from SIDIS at higher values of $Q^{2}$. Thus, in addition to expanding the current experimental kinematic sensitivity, the STAR data should provide the opportunity to probe theoretical questions such as TMD factorization-breaking, universality, and evolution.

\section{References}

[1] J. Ralston and D. Soper, Nucl. Phys. B 152, 109 (1979); R. L. Jaffe and X. Ji, Phys. Rev. Lett. 67, 552 (1991).

[2] B. I. Abelev et al. (STAR Collaboration), Phys. Rev. Lett. 101, 222001 (2008).

[3] G. Kane, J. Pumplin, and W. Repko, Phys. Rev. Lett. 41, 1689 (1978).

[4] A. Efremov and O. Teryaev, Yad. Fiz. 36, 242 (1982), [Sov. J. Nucl. Phys. 36, 140 (1982)].

[5] J. Qiu and G. Sterman, Phys. Rev. D 59, 014004 (1998).

[6] D. Sivers, Phys. Rev. D 41, 83 (1990); 43, 261 (1991).

[7] K. Kanazawa and Y. Koike, Phys. Lett. B 720, 161 (2013); K. Kanazawa, Y. Koike, A. Metz, and D. Pitonyak, Phys. Rev. D 89, 111501 (2014).

[8] J. Collins, Nucl. Phys. B 396, 161 (1993).

[9] D. Boer, P. J. Mulders, and F. Pijlman, Nucl. Phys. B 667, 201 (2003).

[10] A. Airapetian et al. (HERMES Collaboration), Phys. Lett. B 693, 11 (2010); M. G. Alekseev et al. (COMPASS Collaboration), ibid. 692, 240 (2010); X. Qian et al. (Jefferson Lab Hall A Collaboration), Phys. Rev. Lett. 107, 072003 (2011).

[11] R. Seidl et al. (Belle Collaboration), Phys. Rev. Lett. 96, 232002 (2006); Phys. Rev. D 86, 039905(E) (2012); J. P. Lees et al. (BaBar Collaboration), ibid. 90, 052003 (2014).

[12] M. Anselmino et al., Phys. Rev. D 87, 094019 (2013); Z.-B. Kang et al., arXiv:1505.05589.

[13] U. D’Alesio, F. Murgia, and C. Pisano, Phys. Rev. D 83, 034021 (2011).

[14] M. Anselmino et al., Phys. Rev. D 73, 014020 (2006).

[15] A. Bacchetta and M. Radici, Phys. Rev. D 70, 094032 (2004).

[16] A. Vossen et al. (Belle Collaboration), Phys. Rev. Lett. 107, 072004 (2011).

[17] T. C. Rogers and P. J. Mulders, Phys. Rev. D 81, 094006 (2010).

[18] K. H. Ackermann et al., Nucl. Instr. Meth. A499, 624 (2003), and references therein.

[19] R. Fatemi (STAR Collaboration), AIP Conf. Proc. 1441, 233 (2012).

[20] L. Adamczyk et al. (STAR Collaboration), arXiv:1504.00415.

[21] A. Mukherjee and W. Vogelsang, Phys. Rev. D 86, 094009 (2012).

[22] W. J. Llope et al., Nucl. Instr. Meth. A759, 23 (2014).

[23] M. Cacciari, G. P. Salam, and G. Soyez, J. High Energy Phys. 04, 063 (2008). 
[24] J. L. Drachenberg (STAR Collaboration), EPJ Web Conf. 73, 02009 (2014).

[25] J. L. Drachenberg (STAR Collaboration), in Proceedings of the 20th Particles and Nuclei International Conference (PANIC2014) (2014).

[26] J. K. Adkins and J. L. Drachenberg (STAR Collaboration), in Proceedings of the 21st International Symposium on Spin Physics (Spin2014) (2015).

[27] M. J. Skoby (STAR Collaboration), in Proceedings of the 21st International Symposium on Spin Physics (Spin2014) (2015).

[28] K. Landry (STAR Collaboration), Measuring transversity in $P^{\uparrow}+P$ Collisions with Di-Hadron Correlations at $\sqrt{S}=200 \mathrm{GeV}$ at the STAR Experiment, APS April Meeting 2015.

[29] E. C. Aschenauer et al., The RHIC SPIN Program: Achievements and Future Opportunities (2015), arXiv:1501.01220.

[30] L. Adamczyk et al. (STAR Collaboration), Phys. Rev. D 86, 032006 (2012). 